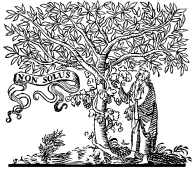

ELSEVIER

\title{
Dogs in society can prevent society going to the dogs
}

It has been suggested that there is no such thing as 'human society' as the culture in which we live is naturally a multispecies one. The word 'society' is derived from the Latin societas, and was used to refer to a fellowship or group of individuals with a common interest. In this sense it might be reasonably argued that working and pet dogs are an integral part of a society shared with us.

Clearly dogs and humans have had a particularly close relationship for many thousands of years and indeed it has been suggested that the partnership is derived from co-evolution between the two species (Schleidt and Shalter, 2003). Dogs certainly show a remarkable ability to read a range of human communicative gestures such as gaze orientation (Gácsi et al., 2004) and pointing (Soproni et al., 2002) and it is increasingly argued that such abilities were instrumental to the evolution of the dog (Hare et al., 2002; Miklósi, 2007). Humans also appear intuitively to understand some aspects of dog behaviour, such as certain forms of vocalisation (Molnár et al., 2006). But communication between the two species is not without difficulty at times, as the data on dog bite injuries, as is presented by Belen Rosado and colleagues in this issue of The Veterinary Journal (Rosado et al., 2008).

Dogs are not however malicious, and aggression is generally not a response without good cause, thus each bite represents a failure in communication and a failing of our society. It is worth noting that in the study reported here, only $3 \%$ of bites were from supposedly 'dangerous breeds' and different groups of people appear to be at different levels of risk, so this is clearly not simply a dog problem that needs to be addressed but rather one relating to human-dog interaction.

Accordingly, preventive strategies need to be aimed at both human and dog behaviour and education. In order to develop effective preventive programmes it is essential to recognise the importance of data relating to the circumstances leading to a dog bite injury. Unfortunately this appears to be particularly difficult to obtain; in the current study (Rosado et al., 2008), the circumstances were only recorded in 169/4186 bite incidents, which equates to a $4 \%$ level of reporting. While this may seem to be a relatively straightforward exercise, future research will need to iden- tify why this information is difficult to obtain; even measures aimed at increasing staff motivation to record such data have generally failed to improve success (Bernardo et al., 2002).

In the interim we should aim to increase education about dog behaviour, since the average school child seems to know more about dinosaurs and fictitious creatures than those with which it may interact on a daily basis. Understanding dog behaviour can also help us understand our own behaviour better and so may benefit society in other ways. With this in mind, an educational programme is currently being developed in the UK $(\mathrm{K}$. Shepherd, personal communication), using aggression in dogs as a model to explain to school children why people might become aggressive and the importance of good communication in its prevention. There is also growing interest in pet dog keeping in China, (a country where until recently advertising pet food was illegal), since the government there too appear to have identified the value of dogs in developing human social and communicative skills - an obvious priority for a country with a one child family policy.

Another feature of society is the implied social contract, in which individuals surrender certain freedoms in order to maintain a relationship. In this respect, dogs appear to be good members of our society. They are renowned for their faithfulness and even selectively respond to human direction when it contradicts their own sense of smell (Szetei et al., 2003); there is also a growing list of recognised physical and psychological benefits that come from dog ownership and interaction. By contrast, humans as a species appear more willing to surrender their responsibility to society as statistics relating to the turnover in pet dog ownership show (Patronek and Rowan, 1995).

Perhaps when we appreciate how much dogs are genuinely an integral part of our society we will be more prepared and willing to seek out solutions of mutual benefit to both species rather than simplify the problem to the level of self-interest. Confucius said: 'Consideration for others is the basic of a good life, a good society', and current evidence suggests that we should include dogs in this consideration. 


\author{
Daniel S. Mills \\ Head of Department of Biological Sciences, \\ University of Lincoln, \\ Riseholme Park, \\ Lincoln, LN2 2LG, UK \\ E-mail address: dmills@lincoln.ac.uk \\ Tiny De Keuster \\ Oostvelkouter 222, \\ B-9920 Lovendegem, Belgium
}

E-mail addresses: tiny.dekeuster@telenet.be

\section{References}

Bernardo, L.M., Gardner, M.J., O’Dair, J., Cohen, B., Lucke, J., Pitetti, R., 2002. The DOG BITES program: documentation of growls and bites in the emergency setting. Journal of Emergency Nursing 28, 536541.

Gácsi, M., Miklósi, A., Varga, O., Topál, J., Csányi, V., 2004. Are readers of our face readers of our minds? Dogs (Canis familiaris) show situation-dependent recognition of human's attention. Animal Cognition 7, 144-153.

Hare, B., Brown, M., Williamson, C., Tomasello, M., 2002. The domestication of social cognition in dogs. Science 298, 1634-1636.

Miklósi, Á., 2007. Dog Behaviour, Evolution and Cognition. Oxford University Press, Oxford.

Molnár, Cs., Pongrácz, P., Dóka, A., Miklósi, Á., 2006. Can humans discriminate between dogs on the base of the acoustic parameters of barks? Behavioural Processes 73, 76-83.

Patronek, G.J., Rowan, A.N., 1995. Determining dog and cat numbers and population dynamics. Anthrozoos 8, 199-205.

Rosado, B., Garcia-Belenguer, S., Leon M., Palacio J., 2008. A comprehensive study of dog bites in Spain, 1995-2004. The Veterinary Journal.

Schleidt, W.M., Shalter, M.D., 2003. Co-evolution of humans and canids: an alternative view of dog domestication: Homo homini lupus? Evolution and Cognition 9, 57-72.

Soproni, K., Miklósi, Á., Topál, J., Csányi, V., 2002. Dogs’ responsiveness to human pointing gestures. Journal of Comparative Psychology 116, $27-34$.

Szetei, V., Miklosi, A., Topal, J., Csanyi, V., 2003. When dogs seem to lose their nose: an investigation on the use of visual and olfactory cues in communicative context between dog and owner. Applied Animal Behaviour Science 83, 141-152.
107

108

109

110

111

112

113

114

115

116

117

118

119

Q1 120

121

122 